\title{
On Obtaining Sharp Bounds of the Rate of Convergence for a Class of Continuous-Time Markov Chains
}

\author{
A. I. Zeifman* Y. A. Satinł K. M. Kiseleva ${ }^{\ddagger}$
}

\begin{abstract}
We study inhomogeneous continuous-time weakly ergodic Markov chains with a finite state space. We introduce the notion of a Markov chain with the regular structure of an infinitesimal matrix and study the sharp upper bounds on the rate of convergence for such class of Markov chains.

Keywords. continuous-time Markov chains; inhomogeneous Markov chains; sharp upper bounds; rate of convergence; regular structure; queueing models.
\end{abstract}

\section{Introduction}

As is was noted in (Zeifman and Korolev, 2015), the problem of finding sharp bounds on the rate of convergence to the limiting characteristics for Markov chains is important for at least two following reasons:

(i) usually, it is easier to calculate the limiting characteristics of a process than to find the exact distribution of its state probabilities. Therefore, it is very important to have a possibility to replace the exact distribution by the limiting one (see, for instance, (Chakravarthy, 2017; Di Crescenzo et al. 2016)). In order to be able to determine the time instant starting from which such replacement is possible, one must have sharp bounds for the rate of convergence;

(ii) perturbation bounds play significant role in applications. It is well known that even for homogeneous chains the best bounds require the corresponding best bounds on the rate of convergence (Kartashov, 1985, 1996; Liu, 2012; Mitrophanov, 2003, 2004). Bounds for general inhomogeneous Markov chains are also based on the estimates of the rate of convergence in the special weighted norms (Zeifman and Korolev, 2014).

\footnotetext{
*Vologda State University; Institute of Informatics Problems, Federal Research Center "Computer Science and Control", Russian Academy of Sciences; Vologda Research Center of the Russian Academy of Sciences; e-mail a_zeifman@mail.ru

${ }^{\dagger}$ Vologda State University; e-mail yacovi@mail.ru

${ }^{\ddagger}$ Vologda State University; e-mail ksushakiseleva@mail.ru
} 
In (Zeifman and Korolev, 2015) and (Zeifman et al. 2018b) we have studied upper and lower bounds for finite inhomogeneous continuous-time Markov chains. Moreover, in (Zeifman and Korolev, 2015) the new approach to finding sharp upper bounds in natural metrics via essential positivity of the reduced intensity matrix of a Markov chain has been presented. In this paper we determine the class of Markov chains for which this condition (i.e. essential positivity of the reduced intensity matrix; see matrix $B^{*}(t)$ below) is satisfied. It is shown that this class is fairly wide.

Let $X(t)$ be a finite continuous-time Markov chain with infinitesimal matrix $Q(t)=\left(q_{i, j}(t)\right)_{i, j=0}^{S}$ and $A(t)=Q^{T}(t)$ - the transposed infinitesimal matrix of the process, respectively all $a_{i, j}(t)=q_{j, i}(t)$. Denote by $p_{i j}(s, t)=$ $P\{X(t)=j \mid X(s)=i\}, i, j \geq 0,0 \leq s \leq t$ the transition probabilities of $X(t)$ and by $p_{i}(t)=P\{X(t)=i\}$ - the probability that the Markov chain $X(t)$ is in state $i$ at time $t$. Let $\mathbf{p}(t)=\left(p_{0}(t), p_{1}(t), \ldots, p_{S}(t)\right)^{T}$ be the probability distribution vector at instant $t$.

Definition 1 A Markov chain $X(t)$ is called a chain with the regular infinitesimal matrix if $q_{i, i+k}(t)$ and $q_{i+k, i}(t)$ decrease in $k$ for any $t \geq 0$ and any fixed $i$.

Consider the following four classes of finite inhomogeneous continuoustime Markov chains (see also (Zeifman et al. 2018a) and (Zeifman et al. 2018c) for more detailed description).

(I) inhomogeneous birth-death processes, where all $a_{i j}(t)=0$ for any $t \geq 0$ if $|i-j|>1$, and $a_{i, i+1}(t)=\mu_{i+1}(t), \quad a_{i+1, i}(t)=\lambda_{i}(t)$ - birth and death rates respectively;

(II) inhomogeneous chains with "batch" births and single deaths, where $a_{i j}(t)=0$ for any $t \geq 0$ if $i<j-1$, all "birth" rates do not depend on the size of a "population", where $a_{i+k, i}(t)=a_{k}(t)$ for $k \geq 1$ - the rate of "birth" of a group of $k$ particles, $a_{i, i+1}(t)=\mu_{i+1}(t)$ - the death rate; under the additional condition $a_{k+1}(t) \leq a_{k}(t)$ for any positive $k$;

(III) inhomogeneous chains with "batch" deaths and single births, where all $a_{i j}(t)=0$ for any $t \geq 0$ if $i>j+1$, and all "death" rates do not depend on the size of a "population", where $a_{i, i+k}(t)=b_{k}(t), k \geq 1$ is the rate of "death" of a group of $k$ particles, and $a_{i+1, i}(t)=\lambda_{i}(t)$ is the birth rate; under the additional condition $b_{k+1}(t) \leq b_{k}(t)$ for any positive $k$;

(IV) inhomogeneous chains with "batch" births and deaths, where all rates do not depend on the size of a "population", where $a_{i+k, i}(t)=a_{k}(t)$, and $a_{i, i+k}(t)=b_{k}(t)$ for $k \geq 1$ are the rates of "birth" and "death" of a group of $k$ particles respectively, under the additional condition $a_{k+1}(t) \leq a_{k}(t)$ and $b_{k+1}(t) \leq b_{k}(t)$, for any positive $k$. 
One can see that all of the four Markov chains have the regular structure of their infinitesimal matrices.

\section{Transformations for a class of Markov chains with the regular structure of an infinitesimal matrix}

Consider the forward Kolmogorov system of ordinary differential equations for the inhomogeneous Markov chain $X(t)$ in the form

$$
\frac{d}{d t} \mathbf{p}(t)=A(t) \mathbf{p}(t)
$$

Using the substitution $p_{0}(t)=1-\sum_{i=1}^{S} p_{i}(t)$, we obtain from (11) the reduced system in the form

$$
\frac{d}{d t} \mathbf{z}(t)=B(t) \mathbf{z}(t)+\mathbf{f}(t),
$$

where $\mathbf{f}(t)=\left(a_{10}(t), a_{20}(t), \cdots, a_{S 0}(t)\right)^{T}, \mathbf{z}(t)=\left(p_{1}(t), p_{2}(t), \cdots, p_{S}(t)\right)^{T}$,

$$
B(t)=\left(\begin{array}{cccc}
a_{11}(t)-a_{10}(t) & a_{12}(t)-a_{10}(t) & \cdots & a_{1 S}(t)-a_{10}(t) \\
a_{21}(t)-a_{20}(t) & a_{22}(t)-a_{20}(t) & \cdots & a_{2 S}(t)-a_{20}(t) \\
a_{31}(t)-a_{30}(t) & a_{32}(t)-a_{30}(t) & \cdots & a_{3 S}(t)-a_{30}(t) \\
\cdots & & & \\
a_{S 1}(t)-a_{S 0}(t) & a_{S 2}(t)-a_{S 0}(t) & \cdots & a_{S S}(t)-a_{S 0}(t)
\end{array}\right) .
$$

All bounds on the rate of convergence to the limiting regime for $X(t)$ correspond to the same rate of convergence bounds of the solutions of system

$$
\frac{d}{d t} \mathbf{y}(t)=B(t) \mathbf{y}(t)
$$

Denote by $T$ the upper triangular matrix of the form

$$
T=\left(\begin{array}{ccccc}
1 & 1 & 1 & \cdots & 1 \\
0 & 1 & 1 & \cdots & 1 \\
0 & 0 & 1 & \cdots & 1 \\
\vdots & \vdots & \vdots & \ddots & \\
0 & 0 & 0 & \cdots & 1
\end{array}\right)
$$

Multiply (from the left) the left and the right part of (4) by $T$ and put $\mathbf{u}(t)=T \mathbf{y}(t)$. In such a way the system (4) is transformed into the system

$$
\frac{d}{d t} \mathbf{u}(t)=B^{*}(t) \mathbf{u}(t)
$$


where $B^{*}(t)=T B(t) T^{-1}=\left(b_{i j}^{*}(t)\right)_{i, j=1}^{S}$ and the entries $b_{i j}^{*}(t)$ having the following form:

$$
\begin{gathered}
b_{11}^{*}(t)=-a_{01}-a_{10}-a_{20}-\cdots-a_{S 0}, b_{12}^{*}(t)=a_{01}-a_{02}, \\
b_{13}^{*}(t)=a_{02}-a_{03}, b_{14}^{*}(t)=a_{03}-a_{04}, \cdots, b_{1 S}^{*}(t)=a_{0 S-1}-a_{0 S}, \\
b_{21}^{*}(t)=a_{21}-a_{20}+a_{31}-a_{30}+\cdots+a_{S 1}-a_{S 0}, \\
b_{22}^{*}(t)=-a_{02}-a_{12}-a_{21}-a_{31}-\cdots-a_{S 1}, \\
b_{23}^{*}(t)=-a_{13}+a_{12}-a_{03}+a_{02}, \\
b_{24}^{*}(t)=-a_{14}+a_{13}-a_{04}+a_{03}, \cdots, \\
b_{2 S}^{*}(t)=-a_{1 S}+a_{1 S-1}-a_{0 S}+a_{0 S-1}, \\
b_{31}^{*}(t)=a_{31}-a_{30}+a_{41}-a_{40}+\cdots+a_{S 1}-a_{S 0}, \\
b_{32}^{*}(t)=a_{32}-a_{31}+a_{42}-a_{41}+\cdots+a_{S 2}-a_{S 1}, \\
b_{33}^{*}(t)=-a_{03}-a_{13}-a_{23}-a_{32}-a_{42}-\cdots-a_{S 2}, \\
b_{34}^{*}(t)=a_{03}-a_{04}+a_{13}-a_{14}+a_{23}-a_{24}, \cdots, \\
b_{3 S}^{*}(t)=a_{0 S-1}-a_{0 S}+a_{1 S-1}-a_{1 S}+a_{2 S-1}-a_{2 S}, \\
b_{44}^{*}(t)=-a_{04}-a_{14}-a_{24}-a_{34}-a_{43}-a_{53}-\cdots-a_{S 3}, \cdots, \\
b_{S, S}^{*}(t)=-a_{S, S-1}-a_{0 S}-a_{1 S}-a_{2 S}-a_{3 S}-\cdots-a_{S-1, S}, \\
b_{41}^{*}(t)=a_{41}-a_{40}+a_{51}-a_{50}+\cdots+a_{S 1}-a_{S 0}, \\
b_{42}^{*}(t)=a_{42}-a_{41}+a_{52}-a_{51}+\cdots+a_{S 2}-a_{S 1}, \\
b_{S 1}^{*}(t)=a_{43}-a_{42}+a_{53}-a_{52}+\cdots+a_{1 S}+a_{2 S-1}-a_{2 S}+a_{3 S-1}-a_{3 S}, \\
b_{S 2}^{*}(t)=a_{S 2}-a_{S 1}, \\
b_{S 3}^{*}(t)=a_{S 3}-a_{S 2}, \\
\end{gathered}
$$

By inspecting the values of $b_{i j}^{*}(t)$ we arrive at the following theorem. 
Theorem 1 Let $X(t)$ be a finite Markov chain with the regular structure of an infinitesimal matrix. Then the corresponding respective matrix $B^{*}(t)=$ $T B(t) T^{-1}$ is essentially non-negative, i.e., $b_{i j}^{*}(t) \geq 0$ for all $i, j$, such that $i \neq j$ and any $t \geq 0$.

Remark 1 One can note that the assumption of regularity of infinitesimal matrix is close to the necessity for essential non-negativity of $B^{*}(t)$. However, there are examples for which infinitesimal matrix is not regular and $B^{*}(t)$ is essential non-negative, see (Ammar and Alharbi, 2018; Dharmaraja, 2000; Zeifman et al. 2019).

\section{General bounds}

In addition to the transformation $T$, described above, we introduce the new diagonal transformation $D=\operatorname{diag}\left(d_{1}, \ldots, d_{S}\right)$, in which all the entries $d_{j}$ are positive. By left-multiplying the left and the right part of (6) by $D$ and putting $\mathbf{w}(t)=D \mathbf{u}(t)=D T \mathbf{y}(t)$, we obtain

$$
\frac{d \mathbf{w}}{d t}=B^{* *}(t) \mathbf{w}(t)
$$

where $B^{* *}(t)=D B^{*}(t) D^{-1}=D T B(t) T^{-1} D^{-1}=\left(b_{i j}^{* *}(t)\right)_{i, j=1}^{S}$ is also essentially non-negative for any $t \geq 0$.

Consider column sums of elements of $B^{* *}(t)$ and the largest and smallest of them:

$$
h^{* *}(t)=\max _{1 \leq k \leq S} \sum_{i} b_{i k}^{* *}(t), \quad h_{* *}(t)=\min _{1 \leq k \leq S} \sum_{i} b_{i k}^{* *}(t) .
$$

Using the same approach, which was used in the Theorem 1 of (Zeifman and Korolev, 2015), we arrive at the following theorem.

Theorem 2 Let $X(t)$ be a finite Markov chain with the regular structure of an infinitesimal matrix. Then for any positive $d_{k}$ the following bounds on the rate of convergence hold:

$$
\|\mathbf{w}(t)\| \leq \exp \left\{\int_{0}^{t} h^{* *}(\tau) d \tau\right\}\|\mathbf{w}(0)\|,
$$

for any corresponding initial condition $\mathbf{w}(0)$, and

$$
\|\mathbf{w}(t)\| \geq \exp \left\{\int_{0}^{t} h_{* *}(\tau) d \tau\right\}\|\mathbf{w}(0)\|,
$$

if the initial condition is non-negative, where $\|\cdot\|$ denotes the $l_{1}$-norm, i.e., $\|\mathbf{x}\|=\sum\left|x_{i}\right|$. 
In the homogeneous case, sharp upper bounds on the rate of convergence can be obtained for all of the fours classes of the considered Markov chains (classes (I)-(IV)).

Firstly note that if a matrix $C$ is essentially non-negative and irreducible then there exists a unique positive diagonal matrix $D=\operatorname{diag}\left(d_{1}, \ldots, d_{S}\right)$ such that all column sums of matrix $C_{D}=D C D^{-1}$ are the same (and are equal to the maximal eigenvalue $\lambda_{0}$ of $C$ ). For the proof one can put $m=$ $\max \left|c_{j j}\right|$ and consider non-negative matrix $C^{\prime}=(C)^{T}+m I$. Matrix $C^{\prime}$ is non-negative and irreducible, hence it has a positive eigenvalue $\lambda^{*}=\lambda_{0}+m$, and the respective eigenvector $\mathbf{x}=\left(x_{1}, \ldots, x_{S}\right)^{T}$ has positive coordinates. Put $d_{k}=x_{k}^{-1}$. Then vector $\mathbf{e}=(1, \ldots, 1)^{T}$ is eigenvector of matrix $C_{D}^{\prime}=$ $D C^{\prime} D^{-1}$, and therefore all row sums for this matrix is equal to $\lambda^{*}$. Then all row sums for matrix $C_{D}^{T}=C_{D}^{\prime}-m I$ is equal to $\lambda^{*}-m=\lambda_{0}$, and every column sum for matrix $C_{D}$ will be the same. This implies an existence of positive diagonal matrix $D=\operatorname{diag}\left(d_{1}, \ldots, d_{S}\right)$, and its uniqueness is evident.

Theorem 3 Let $X(t)$ be a finite homogeneous Markov chain with the regular structure of an infinitesimal matrix. Then there exists a positive sequence $\left\{d_{k}\right\}$ such that $h^{* *}=h_{* *}$, and hence

$$
\|\mathbf{w}(t)\|=\exp \left\{h_{* *} t\right\}\|\mathbf{w}(0)\|,
$$

if the initial condition is non-negative, and if:

(I) $X(t)$ is a birth-death processes with positive rates $\mu_{i+1}$ and $\lambda_{i}$;

(II) $X(t)$ is a Markov chain with 'batch' births and single deaths with positive $\mu_{i+1}$, and $a_{2}<a_{1}$

(III) $X(t)$ is a Markov chain with 'batch' deaths and single births with positive $\lambda_{i}$ and $b_{2}<b_{1}$;

(IV) $X(t)$ is a Markov chain with 'batch' births and deaths with $a_{2}<a_{1}$ and $b_{2}<b_{1}$.

Proof. For the first class we have

$$
B^{*}=\left(\begin{array}{ccccc}
-\left(\lambda_{0}+\mu_{1}\right) & \mu_{1} & 0 & \cdots & 0 \\
\lambda_{1} & -\left(\lambda_{1}+\mu_{2}\right) & \mu_{2} & \cdots & 0 \\
\ddots & \ddots & \ddots & \ddots & \ddots \\
0 & \cdots & \cdots & \lambda_{S-1} & -\left(\lambda_{S-1}+\mu_{S}\right)
\end{array}\right) .
$$

For the second class we obtain

$$
B^{*}=\left(\begin{array}{ccccc}
a_{11}-a_{S} & \mu_{1} & 0 & \cdots & 0 \\
a_{1}-a_{S} & a_{22}-a_{S-1} & \mu_{2} & \cdots & 0 \\
\ddots & \ddots & \ddots & \ddots & \ddots \\
a_{S-1}-a_{S} & \cdots & \cdots & a_{1}-a_{2} & a_{S S}-a_{1}
\end{array}\right) .
$$


For the third class we have

$$
B^{*}=\left(\begin{array}{ccccc}
-\left(\lambda_{0}+b_{1}\right) & b_{1}-b_{2} & b_{2}-b_{3} & \cdots & b_{S-1}-b_{S} \\
\lambda_{1} & -\left(\lambda_{1}+\sum_{i \leq 2} b_{i}\right) & b_{1}-b_{3} & \cdots & b_{S-2}-b_{S} \\
\ddots & \ddots & \ddots & \ddots & \ddots \\
0 & \cdots & \cdots & \lambda_{S-1} & -\left(\lambda_{S-1}+\sum_{i \leq S} b_{i}\right)
\end{array}\right) .
$$

Finally, for the fourth class we have

$$
B^{*}=\left(\begin{array}{ccccc}
a_{11}-a_{S} & b_{1}-b_{2} & b_{2}-b_{3} & \ldots & b_{S-1}-b_{S} \\
a_{1}-a_{S} & a_{22}-a_{S-1} & b_{1}-b_{3} & \cdots & b_{S-2}-b_{S} \\
\ddots & \ddots & \ddots & \ddots & \ddots \\
a_{S-1}-a_{S} & \cdots & \cdots & a_{1}-a_{2} & a_{S S}-a_{1}
\end{array}\right) .
$$

Note that in all cases, all off-diagonal elements of the matrix $B^{*}$ are non-negative, and all elements adjacent to the main diagonal are strictly positive.

Hence under assumptions of Theorem 3 all the matrices $B^{*}$ are essentially non-negative and irreducible, hence all column sums for the corresponding matrices $B^{* *}$ are the same and $h^{* *}=h_{* *}$.

Remark 2 A finite birth-death process with the constant and identical rates of birth $\lambda_{k}=a$ and $\mu_{k+1}=b$ was considered in (Granovsky and Zeifman, 1997, 2005), where both upper an lower sharp bonds were obtained. Namely, the corresponding $D=D^{*}$, sharp $\beta_{*}=\beta^{*}=a+b-2 \sqrt{a b} \cos \frac{\pi}{S+1} \rightarrow$ $(\sqrt{a}-\sqrt{b})^{2}$ as $S \rightarrow \infty$, and $\operatorname{sharp} g^{*}=a+b+2 \sqrt{a b} \cos \frac{\pi}{S+1} \rightarrow(\sqrt{a}+\sqrt{b})^{2}$ as $S \rightarrow \infty$ were found, where $\beta_{*}=\beta^{*}=-h^{*}=-h_{*}$.

Remark 3 The possibility of obtaining explicit and exact estimates of the rate of convergence was previously studied for finite homogeneous birth-death processes, see (Granovsky and Zeifman, 1997, 2000; Van Doorn et al, 2010), as well as for chains of fourth class, see (Zeifman and Korolev, 2015).

Acknowledgement. This research was supported by Russian Science Foundation under grant 19-11-00020. 


\section{References}

Ammar, S. I., Alharbi, Y. F. 2018. Time-dependent analysis for a twoprocessor heterogeneous system with time-varying arrival and service rates. Applied Mathematical Modelling, 54, 743-751.

Chakravarthy, S. R. 2017. A catastrophic queueing model with delayed action. Applied Mathematical Modelling, 46, 631-649.

Dharmaraja, S. 2000. Transient solution of a two-processor heterogeneous system, Math. Comput. Model. 32, 1117-1123.

Di Crescenzo, A., Giorno, V., Nobile, A. G. 2016. Constructing transient birth-death processes by means of suitable transformations. Applied Mathematics and Computation, 281, 152-171.

Granovsky, B. L., Zeifman, A. I. 1997. The decay function of nonhomogeneous birth-death processes, with application to mean-field models. Stochastic Process. Appl. 72, 105-120.

Granovsky, B. L., Zeifman, A. I. 2000. The N-limit of spectral gap of a class of birthdeath Markov chains. Appl. Stoch. Models Bus. Ind. 16(4), 235-248.

Granovsky, B. L., Zeifman, A. I. 2005. On the lower bound of the spectrum of some mean-field models. Theory Probab. Appl. 49, 148-155.

Kartashov, N. V. 1985. Criteria for uniform ergodicity and strong stability of Markov chains with a common phase space, Theory Probab. Appl. 30, $71-89$.

Kartashov, N.V. 1996. Strong Stable Markov Chains. VSP. Kiev: TBiMC, Utrecht.

Liu, Y. 2012. Perturbation bounds for the stationary distributions of Markov chains. SIAM. J. Matrix Anal. Appl. 33, 1057-1074.

Mitrophanov, A. Yu. 2003. Stability and exponential convergence of continuous-time Markov chains. J. Appl. Probab. 40, 970-979.

Mitrophanov, A. Yu. 2004. The spectral gap and perturbation bounds for reversible continuous-time Markov chains. J. Appl. Probab. 41, 1219-1222.

Van Doorn, E. A., Zeifman, A. I., Panfilova, T. L. 2010. Bounds and asymptotics for the rate of convergence of birth-death processes. Th. Probab. Appl. 54, 97-113.

Zeifman, A. I., Korolev, V. Y. 2014. On perturbation bounds for continuoustime Markov chains. Statistics \& Probability Letters, 88, 66-72.

Zeifman, A. I., Korolev, V. Y. 2015. Two-sided Bounds on the Rate of Convergence for Continuous-time Finite Inhomogeneous Markov Chains. Statistics \& Probability Letters, 103, 30-36. 
Zeifman, A., Sipin, A., Korolev, V., Shilova, G., Kiseleva, K., Korotysheva, A., Satin, Y. 2018a. On Sharp Bounds on the Rate of Convergence for Finite Continuous-time Markovian Queueing Models, LNCS, 10672, 20-28.

Zeifman, A. I., Korolev, V. Y., Satin, Y. A., Kiseleva, K. M. 2018b. Lower bounds for the rate of convergence for continuous-time inhomogeneous Markov chains with a finite state space. Statistics \& Probability Letters, 137, 84-90.

Zeifman, A., Razumchik, R., Satin, Y., Kiseleva, K., Korotysheva, A., Korolev, V. 2018c. Bounds on the rate of convergence for one class of inhomogeneous Markovian queueing models with possible batch arrivals and services. Int. J. Appl. Math. Comp. Sci. 28, 141-154.

Zeifman, A., Satin, Y., Kiseleva, K., Korolev, V., Panfilova, T. 2019. On limiting characteristics for a non-stationary two-processor heterogeneous system. Applied Mathematics and Computation, 351, 48-65. 